\title{
HRTEM Study of Austenite and Martensite in Splat-Cooled and Nanoscale thin Film Ni-Al
}

\author{
D. Schryvers, M. Yandouzi, D. Holland-Moritz* and L. Toth** \\ University of Antwerp, RUCA, EMAT, Groenenborgerlaan 171, 2020 Antwerp, Belgium \\ * Institut für Raumsimulation, Linder Höhe, 51140 Köln, Germany \\ ** MFKI, Institute of Thin Films, P.O. Box 76, 1325 Budapest, Hungary
}

\begin{abstract}
Selected results of electron microscopic investigations on the atomic and microstructure of $\mathrm{Ni}-\mathrm{Al}$ austenite and martensite in new types of materials are presented. It is shown that splatcooled material behaves similar as normal homogenised bulk material whereas in evaporated thin films the austenite is strongly stabilised.
\end{abstract}

\section{INTRODUCTION}

These days new types of materials of conventional alloys are investigated in view of their martensitic and shape memory characteristics. For Ni-Al the martensitic behaviour in the bulk material has been properly documented by several experimental as well as theoretical studies (for a recent review see [1]). In order to obtain new insight in the influence of the existing defects and the dimensions of the material on the atomic and microstructures occurring during this transformation, $\mathrm{Ni}-\mathrm{Al}$ splat-cooled material and nanoscale thin films were investigated by conventional and high resolution transmission electron microscopy (CTEM and HRTEM). In the first case the rapid quench from the melt yields a very fast crystallisation process which implies the existence of a higher number of point and other defects when compared with the conventional arc-melting procedure used to obtain bulk material. Due to the very short quenching times, splats are usually very homogenous with respect to compositional variation on a macroscopic scale but they are also crystallised in many small grains. For the thin films, the nanoscale dimension of the thickness presents an opportunity to investigate the influence of an anisotropic environment.

\section{SPLAT-COOLED MATERIAL}

Samples with a Ni content of 62.5 and 65 at.\% Ni were splat-cooled at a rate of $5.10^{5} \mathrm{~K} / \mathrm{s}$. During the splatcooling a droplet of the melt is pressed to a thin film by two Cu pistons that are shot against each other. During the contact of the pistons with the melt heat is transferred from the melt into the pistons causing the melt to solidify. The resulting discs have a diameter of approximately $20 \mathrm{~mm}$ and a thickness of about $200 \mu \mathrm{m}$. From these $3 \mathrm{~mm}$ EM samples were cut with their normal parallel with the normal to the splatcooled discs. These samples were then further thinned by the double-jet electropolishing technique using the conventional solution and parameters for bulk Ni-Al [2].

In splat-cooled material of 62.5 at.\% Ni the majority of the grains are a few micron in diameter and consist completely of the B2 phase. Adjacent grains are often observed to have a very similar orientation. This is shown in the overview and SAED patterns in fig. 4 of ref. [3]. All investigated B2 grains are in the precursor state which is clear from the typical $\langle 110\rangle$ diffuse intensity streaks in reciprocal space shown in the inset of fig. 1. The distorted domains in this precursor state do not, however, reveal the micromodulation observed in bulk material of this composition [2]. Indeed, from the HRTEM image of fig. 1 a contiguous configuration of distorted domains, each with an averaged diameter of approximately 2-3 nm, plus strong strain contrast is observed. This observation is similar to the one in bulk material with a higher Ni content $[4,5]$. In the domains revealing a well resolved dot pattern the shear distortion occurs on the $<110\rangle$ planes edge-on in this orientation and can be seen as a displacement of the dot rows when viewing the image along a grazing 110 or 110 incidence. Depending on the actual location the B2 ordering or the basic lattice is visible. The exact origin of this strong difference at such a local scale is unclear. It could be an imaging effect because of different thicknesses or it could indicate microscopic inhomogeneities in the ordering. In some regions a line instead of a dot pattern is observed. When these lines are parallel with the $\langle 100\rangle$ directions in the image plane, as was the case for the bulk examples [4,5], this feature is explained by shear 
planes inclined with respect to the electron beam which yields imperfect atom columns along the viewing direction. However, in the present splat-cooled material these lines are often parallel with the $\langle 110\rangle$ directions in the image. In this case they are probably due to severe internal distortions as a result of the many dislocations observed in CTEM images. Because of the strain contrast and the local changes in the HRTEM image these dislocations are difficult to recognise in these high magnification images. The lack of the micromodulation corresponds with the missing of any intensity enhancements or satellites in the diffuse intensity streaks in reciprocal space.

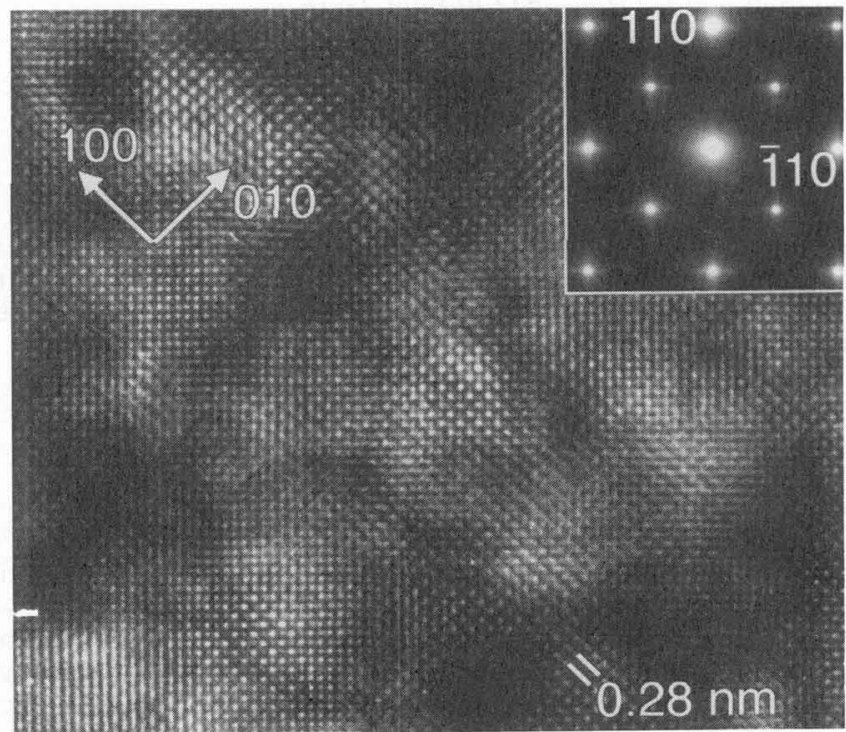

Figure 1. [001] HRTEM image of the precursor domains in a $\mathrm{B} 2$ grain of splat-cooled $\mathrm{Ni} 62.5 \mathrm{Al} 37.5$. The inset shows the typical $<110\rangle$ diffuse intensity streaks around the Bragg reflections.

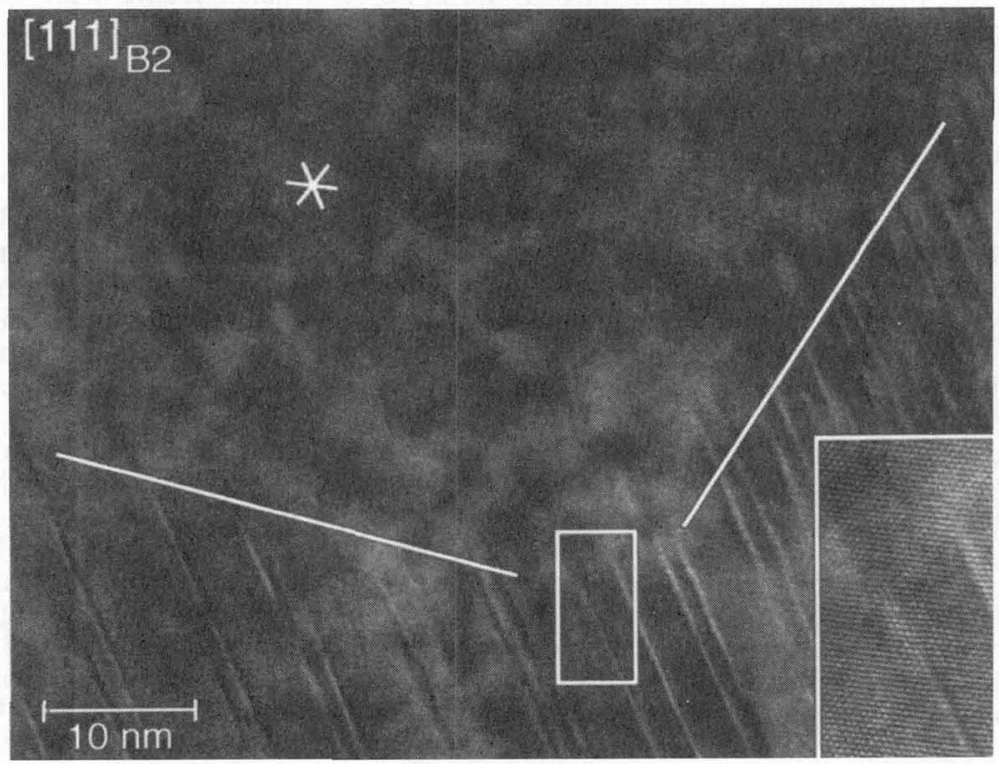

Figure 2. Low magnification HRTEM image of an austenite-martensite interface in splat-cooled $\mathrm{Ni} 62.5 \mathrm{Al} 37.5$ showing a change of habit plane. The star indicates the orientations of the $\{110\}$ families of planes in the B2 area observed along a [111] direction. The inset shows a magnification of the rectangular area revealing the atomic resolution near the interface. 
Some of these grains are partially transformed into the microtwinned $\mathrm{L} 1_{0}$ martensite, occasionally revealing a stacking close to that of the $14 \mathrm{M}$ structure $[2,6]$. Due to the smallness of the grains and the fact that the transformation is apparently caught midway the hysteresis different interfacial configurations can be investigated. In fig. 2 an example of a martensite-austenite interface is shown. The B2 austenite is observed along its [111] direction with one of the edge-on $\{110\}$ families of planes being transformed into the close packed planes of the martensite. In the latter the volume ratio of the large versus small twin variant is rather extreme. From the star in the B2 region indicating the $\{110\}$ families of planes the habit plane of the interface can be seen to change from close to $(110)$ to close to $(01 \overline{1})$. From the magnification in the inset it is seen that the orientation of the (101) planes becoming the twin planes of the martensite hardly changes over the interface. Also no dislocations are observed along this viewing direction. The strongest distortions occur on those planes being differently affected by the different twin variants which leads to some local distortion contrast at the tips of the smallest twin variants.

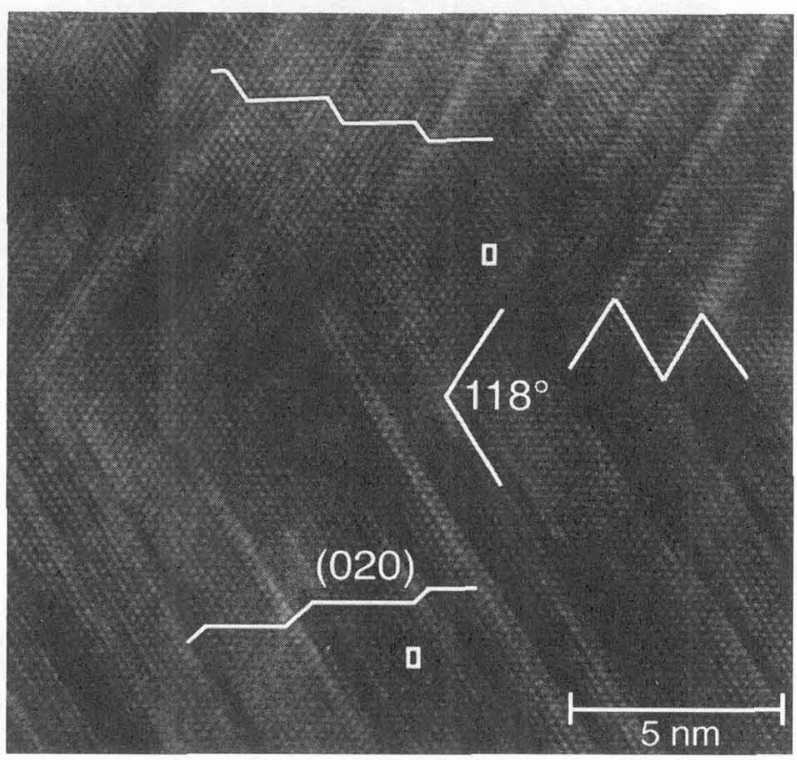

Figure 3. [101] HRTEM image of an interface between two martensite plates in splat-cooled Ni62.5Al37.5. The stacking sequences on both sides are slightly different. The interface follows a zig-zag path as indicated in the right part. Projected unit cells are indicated on both sides as well as the twin effect on the (020) planes.

Fig. 3 shows a macrotwin interface between two martensite plates with a relatively short stacking sequence indicated on the image. This sequence, however, is not very well defined which could be due to the incomplete situation of the transformation. From this image it can be concluded that the interface is parallel with the $(020)$ plane of the largest microtwin variants on both sides and which used to be a (110) plane of the austenite. At most locations the microtwin variants of one given plate stop at a microtwin of the other plate which yields a zig-zag shaped interface. The angle between both microtwin planes is $118^{\circ}$, originating from $120^{\circ}$ between the (110) planes in the austenite [7].

Samples with a 65 at.\% $\mathrm{Ni}$ content are nearly completely transformed to microtwinned martensite, usually as one microtwinned plate within a given grain but sometimes as self-accommodating groups. Some examples are shown in fig. 5 of ref. [3]. Occasionally indications of an irregular 14M-like structure are found. An example of the latter at a boundary with a normally microtwinned plate is shown in fig. 4 with both plates observed along the $[110]_{\mathrm{L} 10}$ direction. The interface is viewed edge-on and perpendicular to the microtwin planes of the 14M plate. From a lower magnification (not shown) it is also seen to be close to parallel with the microtwin planes of the larger plate which are not visible but parallel with the horizontal close packed planes of this variant. In the present case the tips of the thinnest microtwins of the 14M-like stacking reveal more strain contrast than in fig. 2 but again no dislocations are observed in this projection. The microtwin planes do, however, exhibit a severe curvature of approximately $5^{\circ}$ when crossing the boundary and becoming non-twinned close packed planes in the other plate, as indicated on fig. 4 . The fact that the interface is not a symmetry plane for the configuration of both plates indicates that it was formed by coalescence and not by simultaneous growth. 
In plates with wider microtwin variants irregular arrangements of microtwins exist due to specific interfaces between adjacent B2 grains. An example of such a configuration is shown in fig. 6 of ref. [3].

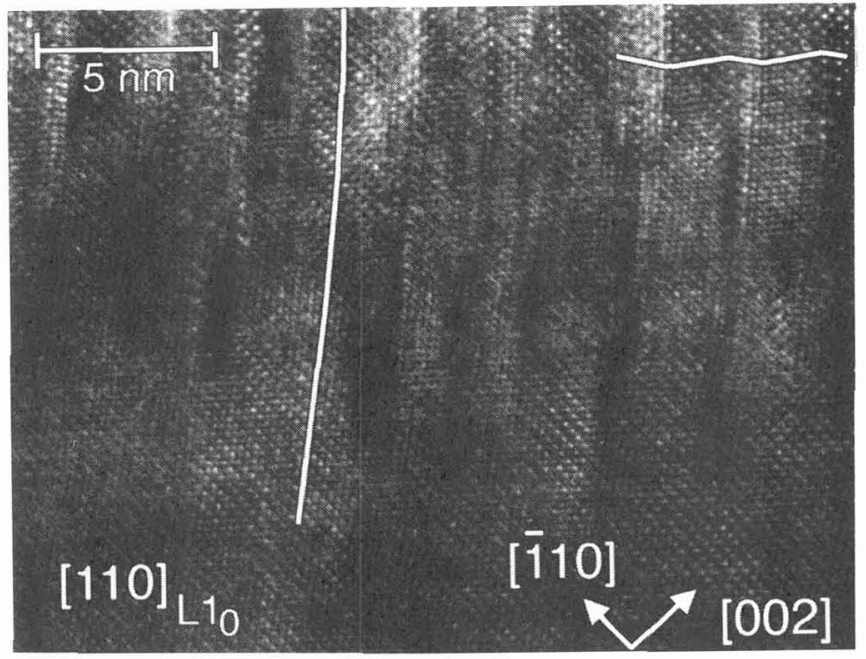

Figure 4. HRTEM image of a martensite-martensite interface in splat-cooled $\mathrm{Ni}_{65} \mathrm{Al}_{35}$ showing strain contrast at the tips of the smallest twins. Severe curvature of the close packed planes is noted.

\section{NANOSCALE THIN FILMS}

Until now most studies of martensitic transformations in thin metallic films only considered films with a thickness in the order of microns. In this case the microscopic behaviour of the film still follows the normal bulk conditions. However, when the film thickness is decreased to a few nanometers the anisotropic nature of the film dimensions can play an important role in the martensitic transformation. Indeed, several investigators have already noted difficulties for transforming a thinned bulk sample in-situ. Also the increased number of lattice defects when compared with well homogenised bulk material will influence this transformation.

In the present study the aim is to produce monophase Ni-Al austenite films with a thickness in the nanometer range which can directly be investigated by the different EM modes. Two compositions, 62.5 and 65 at. \% Ni (further referred to as $62.5 \mathrm{Ni}$ and $65 \mathrm{Ni}$ ), are currently being used. In the bulk the $\mathrm{M}_{\mathrm{S}}$ temperature of $62.5 \mathrm{Ni}$ is reported to be between $-100^{\circ} \mathrm{C}$ and $0^{\circ} \mathrm{C}$ while that for $65 \mathrm{Ni}$ is around $400^{\circ} \mathrm{C}$ $[8,9]$. The evaporation source can be an alloy with this composition or a combination of separate $\mathrm{Ni}$ and $\mathrm{Al}$ sources with the proper ratio. In the latter case both elements are always evaporated simultaneously. Typical evaporation rates are a few $\AA / s$ and films of a few tens of nanometers are obtained. As substrate single crystals of ex-situ cleaved (001) $\mathrm{NaCl}$ and $\mathrm{KCl}$ and which can be heated during deposition up to $550^{\circ} \mathrm{C}$ are used. $\mathrm{NaCl}$ presents the best epitaxial configuration for the preferred $\mathrm{B} 2$ structure but $\mathrm{KCl}$ allows for the highest substrate temperature. The vacuum of the evaporation instrument is within the $10^{-5} \mathrm{mbar}$ range. After deposition the films are floated of by dissolving the substrate in water and then placed on a Cu EM grid.

In fig. 5 a series of SAED patterns comparing the different films prepared under slightly different conditions are presented. From the ring nature of these patterns it is immediately clear that most films consist of very small grains while the actual appearing structures can easily be concluded from the measured ring diameters and ratios between those. In practise the structures of the $\mathrm{B} 2$ and $\mathrm{L}_{2}$ phases are recognised depending on the actual evaporation and substrate conditions. For convenience a schematic of the observed rings is included in fig. 5a.

When comparing the experimental ring patterns with the schematic in fig. $5 \mathrm{a}$, it is clear that in fig. $5 \mathrm{~b}$ both ring systems exist while all other patterns only show the B2 phase. Comparing this with other results it is concluded that the main parameter here is the substrate temperature. Thus, if one wants to start with a monophase film the substrate temperature should not exceed $400^{\circ} \mathrm{C}$. It is also clear that the best epitaxy is obtained in fig. $5 \mathrm{~d}$ which corresponds with the fact that the $\mathrm{NaCl}$ cubic lattice parameter only has a discrepancy of approximately $2 \%$ with that of the B2, whereas this discrepancy becomes about $8 \%$ for $\mathrm{KCl}$. As a result the substrate-film correlation usually becomes $(001) / /(001),[100] / /[100]$ as seen from the 
most intense reflections in the 110 (second) and 200 (fourth) rings, although some weaker reflections in the 100 ring also reveal the existence of a $(001) / /(001),[100] / /[110]$ epitaxial relationship.

In fig. 6 a HRTEM image of the same film as in fig. 5d is shown. The grains are a few nanometers in diameter and the central one clearly shows the [001] B2 type of lattice image. The edges of the grain are primarily parallel with $(100)$ or (110) planes. Inside the grain the lattice image changes from site to site yielding a domain structure which can be compared with that of the precursor distortions as shown in fig. 1. Again no extra micromodulations as in bulk material of this composition are recognised. As expected from the SAED patterns, $65 \mathrm{Ni}$ films do not show grains of this size.

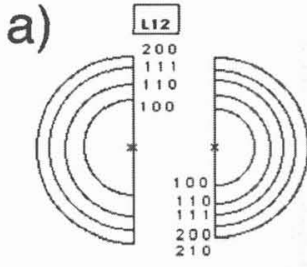

B2

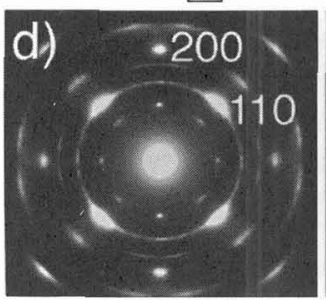

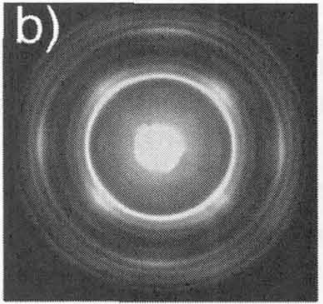

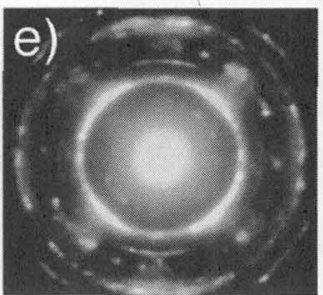

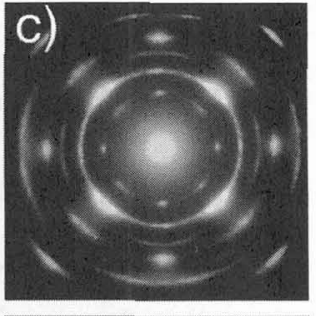

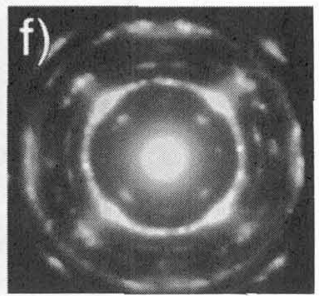

Figure 5. SAED patterns of $\mathrm{Ni}-\mathrm{Al}$ films evaporated under different conditions: (a) schematic of $\mathrm{B} 2$ and $\mathrm{L} 12$ rings, (b) $62.5 \mathrm{Ni}$ on $\mathrm{KCl}$ at $450^{\circ} \mathrm{C}$, (c) $62.5 \mathrm{Ni}$ on $\mathrm{KCl}$ at $300^{\circ} \mathrm{C}$, (d) $62.5 \mathrm{Ni}$ on $\mathrm{NaCl}$ at $300^{\circ} \mathrm{C}$, (e) $65 \mathrm{Ni}$ on $\mathrm{KCl}$ at $400{ }^{\circ} \mathrm{C}$, (f) $65 \mathrm{Ni}$ on $\mathrm{NaCl}$ at $400^{\circ} \mathrm{C}$.

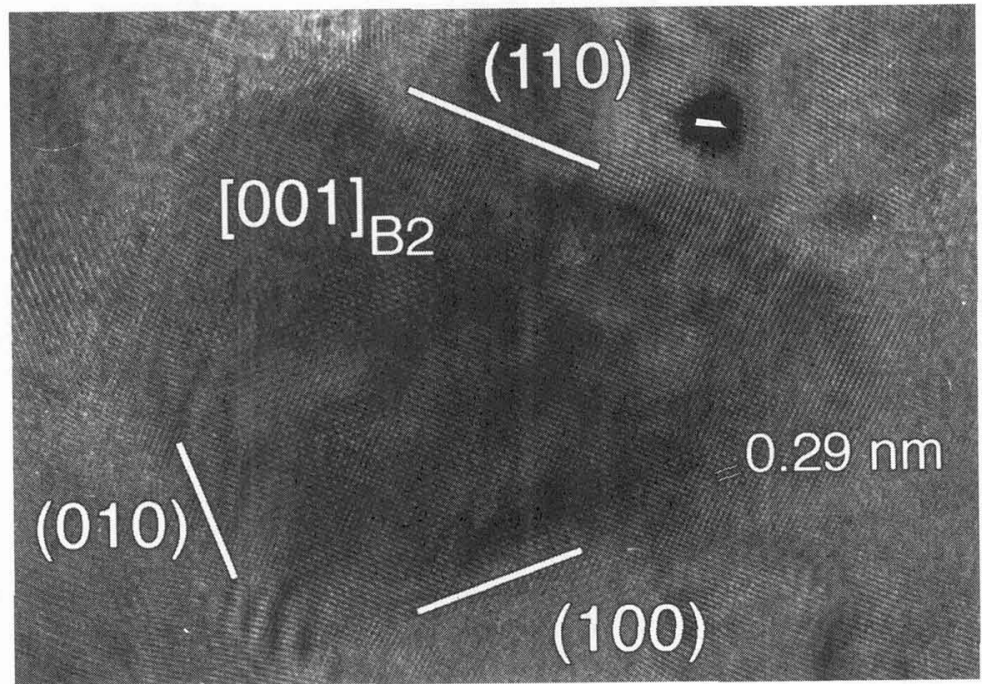

Figure 6. HRTEM image of $62.5 \mathrm{Ni}$ film evaporated onto $\mathrm{NaCl}$ at $300^{\circ} \mathrm{C}$. The [001] zone orientation of the central grain is clearly visible.

When both the $62.5 \mathrm{Ni}$ and $65 \mathrm{Ni}$ films are cooled to $-173{ }^{\circ} \mathrm{C}$ no martensitic transformation was observed, which is clearly different from the bulk transformation. Different reasons for this changed 
behaviour could be suggested. First it is well known that a deposited film can have a composition different from that of the source and knowing the strong dependency of $\mathbf{M}_{\mathrm{S}}$ on composition, this parameter is very important. Different EDX measurement, however, indicate a slight enhancement of $\mathrm{Ni}$ with respect to the source which would in fact increase and not decrease $M_{\mathbf{s}}$. An alternative explanation for the increased stability of the austenite could lie in the increased number of point defects [10] and the increased yield strength of the austenite by grain refinement [11]. Indeed, in view of the low diffusion kinetics at the deposition temperatures, a high density of point defects such as vacancies and antisite atoms can be expected. Also the two-dimensional nature of the film could allow for some extra strain relief without the need for a martensitic transformation.

In an attempt to overcome these austenite stabilising features the $62.5 \mathrm{Ni}$ film was heated in the electron microscope in the hope to enlarge the domains and to induce some atom diffusion to decrease the number of point defects. Unfortunately, in this respect the nanoscale film behaves in a similar way as the bulk material, i.e. it follows the bulk phase diagram. As a result, the film starts to decompose into B2 and $\mathrm{L} 1_{2}$ grains at $750^{\circ} \mathrm{C}$, the latter maintaining a similar epitaxial relationship over large distances. As a result the remaining B2 is further depleted of $\mathrm{Ni}$ and its $\mathrm{M}_{\mathrm{S}}$ drops even further. In fig. 7 the result of such a heating experiment is shown, here after reaching $1000^{\circ} \mathrm{C}$. At higher temperatures the film starts to decompose. From the SAED pattern in fig. 7 it is clear that both structures exist simultaneously and that the $\mathrm{L1}_{2}$ grains indeed reveal the cubic orientation as well yielding a $(001) / /(001),[100] / /[110]$ relation with the B2.

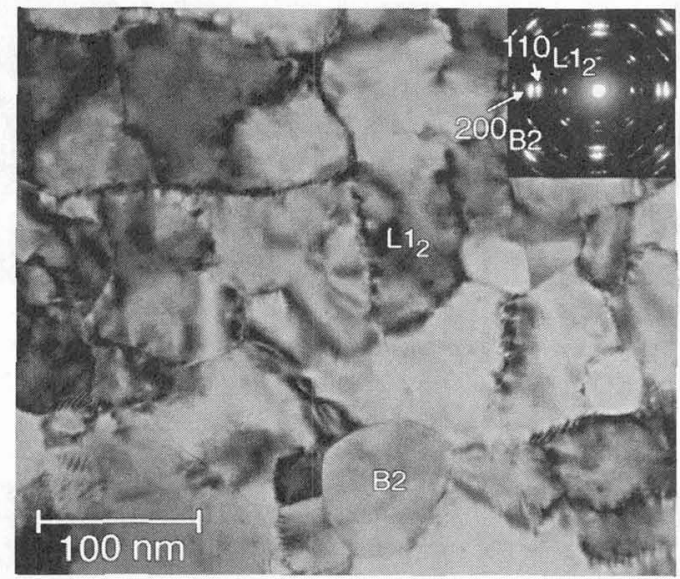

Figure 7. (a) SAED and (b) microstructure of a $62.5 \mathrm{Ni}$ film heated in-situ up to $1000^{\circ} \mathrm{C}$. The formation of well oriented $\mathrm{Ll}_{2}$ grains is clear.

\section{Acknowledgement}

We like to thank Ludo Rossou from the University of Antwerp for help with the thin film preparations and Dr. V. Teodorescu from the Institute of Atomic Physics, Bucharest-Magurele, Romania, for many stimulating discussions on the thin films.

\section{References}

[1] Schryvers D., J. de Physique IV 5 (1995) 225-234.

[2] Schryvers D. and Tanner L.E., Ultramicroscopy 32 (1990) 241-254.

[3] Schryvers D., these proceedings (1997).

[4] Schryvers D., "Experimental studies on precursor phenomena in displacive phase transformations", proc. First Conf. on Alloys, Athens, Greece, 1996, (A. Gonis and P. Turchi, Eds.), (to be publ.).

[5] Schryvers D., habilitation, V.U.B. (1991).

[6] Martynov V.V., Enami K., Khandros L.G., Tkachenko A.V. and Nenno S., Scripta Metall. 17 (1983) 1167-1173.

[7] Baele I., Van Tendeloo G. and Amelinckx S., Acta metall. 35 (1987) 401-412.

[8] Smialek J.L. and Hehemann R.F., Metall. Trans. 4 (1973) 1571-1575.

[9] Au Y.K. and Wayman C.M., Scripta Metall. 6 (1972) 1209-1214.

[10] Murakami Y., Nakajima Y. and Otsuka K., Scripta metall. mater. 34 (1996) 955-962.

[11] Kajiwara S., Metall. Trans. A 17 (1996) 1693-1702. 